\title{
The annual distribution of daily mortality in hospitals and Poisson goodness of fit: a rejoinder
}

\author{
J. P. ZWEIG AND J. Z. CSANK \\ From Sainte Anne's Hospital, Sainte Anne de Bellevue, Province of Quebec, Canada
}

Philippe $^{1}$ has raised an interesting point in regard to the randomness of daily mortality in hospitals. However, his charge, that Zweig and Csank $^{2}$ had concluded only on the basis of their success in fitting a Poisson distribution to the mortality data from large hospitals that 'daily mortality may occur at random and independently ...', is not in accord with what was reported.

The Zweig and Csank description of daily mortality as a discrete random variable follows from the definition of a random variable. It seems obvious also that the essential characteristic of daily mortality, even in hospitals where variations in mortality may occur as a result of seasonal peaks or diurnal changes in hospital care, is that the actual time of occurrence of the death of any particular patient is understandably non-predictable, non-controllable, and independent by nature. This appears to be implicit in the position also adopted in other mortality studies that were referred to, such as Bortkewitsch, ${ }^{3}$ Thorndike, ${ }^{4}$ and Amato. ${ }^{5}$ To conclude otherwise, as Philippe does, by drawing an analogy using Edwards's ${ }^{6}$ algebraic proposition without demonstrating his case by reference to additional hospital data with a direct bearing on annual mortality distributions is to attempt to score a point at small cost.

Philippe's contention that it is a 'fact that the authors' daily mortality does not follow a Poisson law in small hospitals' begs the question, because this was not admitted by Zweig and Csank. What they stated was that the matching in the small hospitals of the mortality distributions with their Poisson models was regarded by them as suggestive of agreement with the Poisson models provided that the less exacting criteria of Cochran ${ }^{7}$ were employed. In fact it was shown that the means and variances of the annual distributions of daily mortality in the smaller hospitals were on the whole as nearly equal as they were in the larger ones and the conformity with their respective Poisson models was acceptable in 30 of the 33 samples involved.

It would be beneficial to see this study extended to other hospitals to find whether the conclusions represent a generalisation which can be upheld. At least evidence from actual observations should be presented and conclusions drawn on the basis of actual data rather than from theory which may or may not apply. Until anyone can show from observed data that the annual distribution of daily mortality in hospitals does not generally conform to a Poisson distribution we believe that the finding that it does so must stand. In this connection it is useful to remember Rothman's ${ }^{8}$ admonition that 'it is better to observe and test in the clinic and laboratory than write polemics concerning one's own metaphors'.

We thank Dr. Y. H. Wang, Department of Mathematics, Concordia University, Montreal, Canada.

Reprints from J. Z. Csank, Chief Psychologist and Director of Psychological Research, Sainte Anne's Hospital, 305 St. Pierre Street, Sainte Anne de Bellevue, Quebec, Canada H9X 1 Y9.

\section{References}

${ }^{1}$ Philippe P. Poisson goodness of fit and the non-randomness of daily mortality. $J$ Epidemiol Community Health 1979; 33: 163.

${ }^{2}$ Zweig JP, Csank JZ. The application of a Poisson model to the annual distribution of daily mortality at six Montreal hospitals. J Epidemiol Community Health 1978; 32: 206-11.

${ }^{3}$ Bortkewitsch L von. Das Gesetz der Kleinen Zahlen. Leipzig: Teubner, 1898. (Also in Thorndike F, op cit, p. 622).

-Thorndike F. Applications of Poisson's probability summation. Bell System Technical Journal 1926; 5: 604-24.

${ }^{5}$ Amato V. L'esponenziale di Poisson e la distribuzione del numero dei morti per giorno. Statistica (Bologna) 1959; 19: $20-59$.

${ }^{6}$ Edwards AWF. The meaning of binomial distribution. Nature 1960; 186: 1074.

${ }^{7}$ Cochran WG. Some methods for strengthening the common $\chi^{2}$ tests. Biometrics 1954, 10: 417-51.

${ }^{8}$ Rothman T, ed. Changing Patterns in Psychiatric Care. Los Angeles: Rush Research Foundation, 1970 and New York: Grown Publishers, 1970: xxi. 